\title{
The Exponentiated Lomax Distribution: Different Estimation Methods
}

\author{
Hamdy M. Salem* \\ Department of Statistics, Faculty of Commerce, Al-Azher University, Egypt \& Community College in Buraidah, Qassim University, \\ Saudi Arabia \\ *Corresponding author: d.hamdysalm@yahoo.com
}

Received September 09, 2014; Revised November 07, 2014; Accepted November 11, 2014

\begin{abstract}
This paper concerns with the estimation of parameters for the Exponentiated Lomax Distribution ELD. Different estimation methods such as maximum likelihood, quasi-likelihood, Bayesian and quasi-Bayesian are used to evaluate parameters. Numerical study is discussed to illustrate the optimal procedure using MATHCAD program (2001). A comparison between the four estimation methods will be performed.
\end{abstract}

Keywords: Exponentiated Lomax Distribution, maximum likelihood estimation, quasi-likelihood estimation, bayesian estimation, quasi-bayesian estimation

Cite This Article: Hamdy M. Salem, "The Exponentiated Lomax Distribution: Different Estimation Methods." American Journal of Applied Mathematics and Statistics, vol. 2, no. 6 (2014): 364-368. doi: 10.12691/ajams-2-6-2.

\section{Introduction}

Authors of the statistical distributions field have continuous motivations for developing a variety distributions to become more flexible and more fitting for real data sets. These new statistical distributions are used to describe and interpret the phenomena. The idea of exponentiated distributions was utilized to create new distributions. Cordeiro \& Castro (2011) extended many known distributions as normal, Weibull, gamma, Gumbel, and inverse Gaussian distributions.

Gupta et al. (1998) introduced a class of exponentiated distributions based on cumulative distribution function CDF for the exponential distribution. In a similar manner, Nadarajah and Kotz (2006) proposed the exponentiated gamma and exponentiated Gumbel distributions. Gauss \& Cordeiro (2013) proposed a new method of adding two parameters to a continuous distribution that extends the idea of Nadarajah and Kotz (2006). Alzaatreh et al. (2013) proposed another new method for generating many new distributions. This method is called, the T-X family of distributions. It has a connection between the hazard functions and each generated distribution as a weighted hazard function of the random variable X. Alzaatreh et al. (2013) founded several known continuous distributions to be special cases of these new distributions.

Wedderbum (1974) introduced an important extension of maximum likelihood estimation to get the optimal parameter estimation. This method is called QuasiLikelihood. It is required assumptions about means and variance functions in contrast to the full distributional assumptions of ordinary likelihood. Quasi-Likelihood for an observation $\mathrm{X}$ with mean $\mu$ and variance $V(\mu)$ takes this form:

$$
\begin{aligned}
& \frac{\partial Q(x ; \mu)}{\partial \mu}=\frac{x-\mu}{V(\mu)} \\
& \text { or } Q(x ; \mu)=\int_{0}^{\mu} \frac{x-\mu}{V(\mu)} d \mu+\text { function of } X
\end{aligned}
$$

where, $\mu$ is $E(X)$ and $V(\mu)$ is $V(X)$.

For a sample of size $n$, the quasi-Bayesian estimation is depended on replacing the likelihood function by the natural exponential of the quasi-likelihood function.

This paper is organized as follows: In Section 2, The ELD distribution will be defined. In section 3, different estimation methods will be used such as maximum likelihood, quasi-likelihood, Bayesian and quasi-Bayesian to obtain the estimators of parameters. Section 4 concerns with comparing procedures of the estimators and compares their performances through numerical simulations.

\section{The Exponentiated Lomax Distribution}

The CDF and the probability density function pdf of the ELD respectively, are:

$$
\begin{aligned}
& F(x)=\left[1-(1+\lambda x)^{-\theta}\right]^{\alpha} ; x>0, \theta, \alpha \text { and } \lambda>0 \\
& f(x)=\alpha \theta \lambda\left[1-(1+\lambda x)^{-\theta}\right]^{\alpha-1}(1+\lambda x)^{-(\theta+1)} \\
& x>0, \theta, \alpha \text { and } \lambda>0
\end{aligned}
$$


Note that, when $\lambda=1$, the pdf of the ELD reduces to the Exponentiated Pareto distribution with parameter $(\theta, \alpha)$. Also, when $\lambda=\alpha=1$, the pdf of the ELD reduces to the standard Lomax distribution with one parameter $\theta$.

The survival function and the hazard function of the ELD respectively, take the following forms:

$$
\begin{aligned}
& S(x)=1-\left[1-(1+\lambda x)^{-\theta}\right]^{\alpha} ; x>0, \theta, \alpha, \text { and } \lambda>0 \\
& h(x)=\frac{\alpha \theta \lambda\left[1-(1+\lambda x)^{-\theta}\right]^{\alpha-1}(1+\lambda x)^{-(\theta+1)}}{1-\left[1-(1+\lambda x)^{-\theta}\right]^{\alpha}} ; \\
& x>0, \quad \theta, \alpha, \text { and } \lambda>0 .
\end{aligned}
$$
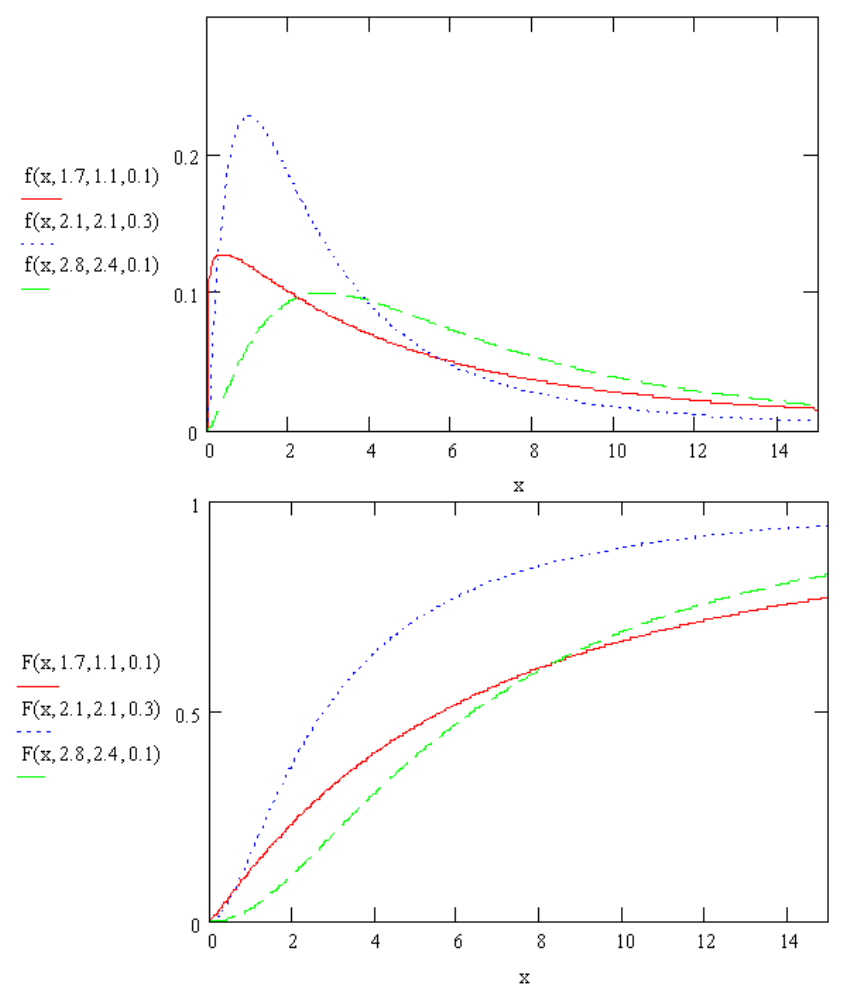

Figure 1. The pdf and CDF carves of the ELD at different values of the parameters $(\theta, \alpha$, and $\lambda)$

The $\mathrm{r}^{\text {th }}$ moments $\mu_{r}^{\prime}$ of the ELD are:

$$
\begin{aligned}
& \mu_{r}^{\prime}=E\left(x^{r}\right)=\alpha \theta \lambda \\
& \int_{0}^{\infty} x^{r}\left[1-(1+\lambda x)^{-\theta}\right]^{\alpha-1}(1+\lambda x)^{-(\theta+1)} d x \\
& =\frac{\alpha}{\lambda^{r}} \sum_{i=0}^{r}\left(\begin{array}{l}
r \\
i
\end{array}\right)(-1)^{i} B\left(1-\frac{1}{\theta}(r-i), \alpha\right) ; r=1,2, \ldots
\end{aligned}
$$

where $B(a, b)=\frac{\mathrm{T}(a) \mathrm{T}(b)}{\Gamma(a+b)}$.

Thus, the mean and variance of the ELD respectively, are:

$$
\mu=\mu_{1}^{\prime}=\frac{\alpha}{\lambda}\left[B\left(1-\frac{1}{\theta}, \alpha\right)-B(1, \alpha)\right],
$$

$$
\operatorname{var}(x)=\left(\frac{\alpha}{\lambda}\right)^{2}\left[\frac{1}{\alpha} B\left(1-\frac{2}{\theta}, \alpha\right)-B^{2}\left(1-\frac{1}{\theta}, \alpha\right)\right]
$$

\section{Different Estimation Methods}

\subsection{Maximum Likelihood Estimators}

The likelihood function of the ELD based on the samples $X_{1}, X_{2}, \cdots, X_{n}$ is:

$$
\begin{aligned}
& L(\lambda, \theta, \alpha) \propto(\alpha \theta \lambda)^{n} \\
& \prod_{i=1}^{n}\left[1-(1+\lambda x)^{-\theta}\right]^{\alpha-1}(1+\lambda x)^{-(\theta+1)}
\end{aligned}
$$

And the log-likelihood functions for $\theta, \alpha$, and $\lambda$ are respectively:

$$
\begin{aligned}
& \ell(\lambda, \theta, \alpha) \propto n[\ln (\lambda)+\ln (\theta)+\ln (\alpha)] \\
& +(\alpha-1) \sum_{i=1}^{n} \ln \left[1-\left(1+\lambda x_{i}\right)^{-\theta}\right]-(\theta+1) \sum_{i=1}^{n} \ln \left(1+\lambda x_{i}\right)
\end{aligned}
$$

The derivatives of (3.1.2) with respect to $\theta, \alpha$, and $\lambda$ respectively, are as follows:

$$
\begin{aligned}
& \frac{\partial \ell}{\partial \theta}=\frac{n}{\theta}+(a-1) \\
& \sum_{i=1}^{n} \frac{\left(1+\lambda x_{1}\right)^{-\theta} \ln \left(1+\lambda x_{1}\right)}{\left[1-\left(1+\lambda x_{i}\right)^{-\theta}\right]}-\sum_{i=1}^{n}\left(1+\lambda x_{1}\right) \\
& \frac{\partial \ell}{\partial \alpha}=\frac{n}{\alpha}+\sum_{i=1}^{n} \ln \left[1-\left(1+\lambda x_{1}\right)^{-\theta}\right] \\
& \frac{\partial \ell}{\partial \lambda}=\frac{n}{\lambda}+\theta(a-1) \\
& \sum_{i=1}^{n} \frac{x_{i}\left(1+\lambda x_{1}\right)^{-(\theta+1)}}{\left[1-\left(1+\lambda x_{i}\right)^{-\theta}\right]}-(\theta+1) \sum_{i=1}^{n} \frac{x_{i}}{\left(1+\lambda x_{i}\right)}
\end{aligned}
$$

The maximum likelihood estimators of the parameters $\theta, \alpha$, and $\lambda$ can be obtained by solving equations (3.1.3), (3.1.4) and (3.1.5) after equating them to zero.

Unfortunately, there is no closed form for the estimators $\hat{\theta}, \hat{\alpha}$, and $\hat{\lambda}$. So, Newton-Raphson method is using to solve these equations in numerical analysis, see Salem (2013).

Now, the log likelihood function which in (3.1.2) can be used to construct Fisher information matrix $I$ has the form:

$$
I^{-1}=\left[\begin{array}{ccc}
-\frac{\partial^{2} \ell}{\partial \theta^{2}} & -\frac{\partial^{2} \ell}{\partial \alpha \partial \theta} & -\frac{\partial^{2} \ell}{\partial \lambda \partial \theta} \\
-\frac{\partial^{2} \ell}{\partial \theta \partial \alpha} & -\frac{\partial^{2} \ell}{\partial \alpha^{2}} & -\frac{\partial^{2} \ell}{\partial \lambda \partial \alpha} \\
-\frac{\partial^{2} \ell}{\partial \theta \partial \lambda} & -\frac{\partial^{2} \ell}{\partial \alpha \partial \lambda} & -\frac{\partial^{2} \ell}{\partial \lambda^{2}}
\end{array}\right]
$$

where, 


$$
\begin{aligned}
& \frac{\partial^{2} \ell}{\partial \theta^{2}}=\left[-\frac{n}{\theta^{2}}+(a-1) \sum_{i=1}^{n}\left[\left(1+\lambda x_{i}\right)^{-\theta} \frac{\ln \left(1+\lambda x_{1}\right)^{2}}{\left[1-\left(1+\lambda x_{i}\right)^{-\theta}\right]}\right.\right. \\
& -\left[\left(1+\lambda x_{i}\right)^{-\theta}\right]^{2} \frac{\ln \left(1+\lambda x_{1}\right)^{2}}{\left[1-\left(1+\lambda x_{i}\right)^{-\theta}\right]^{2}} \\
& \frac{\partial^{2} \ell}{\partial \alpha^{2}}=-\frac{n}{\alpha^{2}} \\
& \frac{\partial^{2} \ell}{\partial \lambda^{2}}=-\frac{n}{\lambda^{2}}+\theta(a-1) \sum_{i=1}^{n}\left[\frac{\left(x_{i}\right)^{2}\left(1+\lambda x_{i}\right)^{-(\theta+1)}(-\theta-1)}{\left(1+\lambda x_{i}\right)\left[1-\left(1+\lambda x_{i}\right)^{-\theta}\right]}\right. \\
& -\frac{\left(x_{i}\right)^{2}\left(1+\lambda x_{1}\right)^{-(\theta+1)}\left(1+\lambda x_{1}\right)^{-\theta} \theta}{\left[1-\left(1+\lambda x_{i}\right)^{-\theta}\right]^{2}} \\
& -(\theta+1) \sum_{i=1}^{n} \frac{\left(x_{i}\right)^{2}}{\left(1+\lambda x_{i}\right)^{2}} \\
& \frac{\partial^{2} \ell}{\partial \theta \partial \alpha}=\sum_{i=1}^{n}\left(1+\lambda x_{1}\right)^{-\theta} \frac{\ln \left(1+\lambda x_{1}\right)}{\left[1-\left(1+\lambda x_{i}\right)^{-\theta}\right]} \\
& \frac{\partial^{2} \ell}{\partial \theta \partial \lambda}=(a-1) \sum_{i=1}^{n} \frac{x_{i}\left(1+\lambda x_{1}\right)^{-(\theta+1)}}{\left[1-\left(1+\lambda x_{i}\right)^{-\theta}\right]} \\
& +\theta(\alpha-1) \sum_{i=1}^{n}\left[-x_{i}\left(1+\lambda x_{1}\right)^{-(\theta+1)}\right. \\
& \times \frac{\ln \left(1+\lambda x_{i}\right)}{\left[1-\left(1+\lambda x_{i}\right)^{-\theta}\right]}-\frac{-x_{i}\left(1+\lambda x_{1}\right)^{-(\theta+1)}}{\left[1-\left(1+\lambda x_{i}\right)^{-\theta}\right]}\left(1+\lambda x_{1}\right)^{-\theta} \\
& \left.\ln \left(1+\lambda x_{i}\right)\right]-\sum_{i=1}^{n} \frac{x_{i}}{\left[1-\left(1+\lambda x_{i}\right)\right]} \\
& \frac{\partial^{2} \ell}{\partial \alpha \partial \lambda}=\theta \sum_{i=1}^{n} \frac{x_{i}\left(1+\lambda x_{1}\right)^{-(\theta+1)}}{\left[1-\left(1+\lambda x_{i}\right)^{-\theta}\right]}
\end{aligned}
$$

\subsection{Quasi-Likelihood Estimators}

Let the pdf of the ELD, $E(x)=\mu$ and $\operatorname{var}(x)$ of the random variable $X$ which is taken from the ELD as in (2.2), (2.6), and (2.7) respectively, then,

$$
\begin{aligned}
& E(x)=\mu=\frac{\alpha}{\lambda}\left[B\left(1-\frac{1}{\theta}, \alpha\right)-B(1, \alpha)\right], \\
\operatorname{var}(x)= & \mu^{2}\left[\frac{1}{\alpha} B\left(1-\frac{2}{\theta}, \alpha\right)-B^{2}\left(1-\frac{1}{\theta}, \alpha\right)\right] \\
= & V(\mu)\left[\frac{1}{\alpha} B\left(1-\frac{2}{\theta}, \alpha\right)-B^{2}\left(1-\frac{1}{\theta}, \alpha\right)\right],
\end{aligned}
$$

where $V($.$) is assumed to be known and the parameter \mu$ may be unknown. So, the Quasi-Likelihood function (1.1) gives:
$Q(x, \theta, \alpha, \lambda)=-\frac{\lambda \cdot \sum_{i=1}^{n} x_{i}}{\alpha\left[B\left(1-\frac{1}{\theta}, \alpha\right)-B(1, \alpha)\right]}$,
$-n\left\{\ln \left(\alpha\left[B\left(1-\frac{1}{\theta}, \alpha\right)-B(1, \alpha)\right]\right)-\ln (\alpha \cdot \lambda)\right\}$,

The derivatives of $Q(x, \mu)$ with respect to $\theta, \alpha$, and $\lambda$ respectively, are:

$$
\begin{aligned}
& \frac{\partial Q}{\partial \theta}=[\Psi(r)-\Psi(k)] \\
& {\left[\frac{n \Gamma(\alpha+1) \Gamma(r)+\sum_{i=1}^{n} x_{i} \lambda \Gamma(k)}{\theta^{2} \Gamma(\alpha+1) \Gamma(r)}\right]} \\
& \frac{\partial Q}{\partial \alpha}=-[1+\alpha \Psi(\alpha)-\alpha \Psi(k)] \\
& {\left[\begin{array}{c}
n \Gamma(\alpha+1) \Gamma(r)-\sum_{i=1}^{n} x_{i} \lambda \Gamma(k) \\
\alpha \Gamma(\alpha+1) \Gamma(r)
\end{array}\right]} \\
& \frac{\partial Q}{\partial \lambda}=-\frac{\sum_{i=1}^{n} x_{i}}{\alpha[\Gamma(r) . s-y]}+\frac{n}{\lambda}
\end{aligned}
$$

where $k=\frac{\theta-1+\alpha \theta}{\theta}, \quad y=\frac{\Gamma(\alpha)}{\Gamma(1+a)}, \quad s=\frac{\Gamma(\alpha)}{\Gamma(k)}$, $r=\frac{\theta-1}{\theta}$, and $\Psi($.$) is the Psi-gamma. It's often called$ Polly-gamma function. For details see (Amos (1983)).

The equations in (3.2.4), (3.2.5), and (3.2.6) will be solved using the same numerical analysis which used in previous maximum likelihood estimation method.

\subsection{Bayesian Estimators}

Let $X_{1}, X_{2}, \cdots, X_{n}$ be independent random samples, drawn from the ELD as equations (2.1), (2.2). The conjugate gamma prior distributions for $\theta, \alpha$ with parameters $(\delta, \beta),(\eta, \varepsilon)$ are employed respectively, as follows:

$$
\begin{aligned}
& g(\theta)=\frac{\beta^{\delta}}{\Gamma(\delta)} \theta^{\delta+1} e^{-\beta \theta} \quad, \theta>0, \quad \delta, \beta>0 \\
& g(\alpha)=\frac{\eta^{\varepsilon}}{\Gamma(\varepsilon)} \alpha^{\varepsilon+1} e^{-\eta \theta} \quad, \theta>0, \quad \varepsilon, \eta>0
\end{aligned}
$$

The non-informative prior distribution of $\lambda$ with parameter $\rho$ is:

$$
g(\lambda)=\rho \quad, \quad 0<\rho>\infty
$$

So, the joint prior distribution for $\theta, \alpha$, and $\lambda$ is: 


$$
g(\theta, \alpha, \lambda)=\frac{\beta^{\delta} \eta^{\varepsilon}}{\Gamma(\delta) \Gamma(\varepsilon)} \rho \theta^{\delta+1} \alpha^{\varepsilon+1} e^{-\eta \theta-\beta \theta}
$$

The posterior density of $\theta, \alpha$, and $\lambda$ Based on the samples $X_{1}, X_{2}, \cdots, X_{n}$ and likelihood function is:

$$
\begin{aligned}
& \pi\left(\Omega \mid X_{1}, X_{2}, \cdots, X_{n}\right)= \\
& \frac{\rho \theta^{\delta+1} \alpha^{\varepsilon+1} e^{-\eta \theta-\beta \theta} L\left(X_{1}, X_{2}, \cdots, X_{n}\right)}{\int_{0}^{\infty} \int_{0}^{\infty} \int_{0}^{\infty} \rho \theta^{\delta+1} \alpha^{\varepsilon+1} e^{-\eta \theta-\beta \theta}}
\end{aligned}
$$

where $\Omega$ is a vector of the parameters $\theta, \alpha$, and $\lambda$, and $L\left(X_{1}, X_{2}, \cdots, X_{n}\right)$ is the likelihood function.

Now, the Bayes estimators of the parameters $\theta, \alpha$, and $\lambda$ under symmetric square loss function can be obtained by getting on the expectation of the marginal distribution of these parameters. In addition, the marginal distribution $h\left(. \mid X_{1}, X_{2}, \cdots, X_{n}\right)$ of any parameter can be obtained by integration of the posterior distribution $\pi\left(\Omega \mid X_{1}, X_{2}, \cdots, X_{n}\right)$ with respect to other parameters. So, the posterior distribution of the parameter $\theta, \alpha$, and $\lambda$ respectively, are:

$$
\begin{aligned}
& h\left(\theta \mid X_{1}, X_{2}, \cdots, X_{n}\right) \\
& =\int_{0}^{\infty} \int_{0}^{\infty} \pi\left(\Omega \mid X_{1}, X_{2}, \cdots, X_{n}\right) d \alpha d \lambda \\
& h\left(\alpha \mid X_{1}, X_{2}, \cdots, X_{n}\right) \\
& =\int_{0}^{\infty} \int_{0}^{\infty} \pi\left(\Omega \mid X_{1}, X_{2}, \cdots, X_{n}\right) d \theta d \lambda \\
& h\left(\lambda \mid X_{1}, X_{2}, \cdots, X_{n}\right) \\
& =\int_{0}^{\infty} \int_{0}^{\infty} \pi\left(\Omega \mid X_{1}, X_{2}, \cdots, X_{n}\right) d \theta d \alpha
\end{aligned}
$$

Consequently, the Bayes estimators of the parameters $\theta, \alpha$, and $\lambda$ under symmetric square loss function respectively, are:

$$
\begin{aligned}
& \tilde{\theta}=E\left[h\left(\theta \mid X_{1}, X_{2}, \cdots, X_{n}\right)\right] \\
& =\int_{0}^{\infty} \theta h\left(\theta \mid X_{1}, X_{2}, \cdots, X_{n}\right) d \theta \\
& \tilde{\alpha}=E\left[h\left(\alpha \mid X_{1}, X_{2}, \cdots, X_{n}\right)\right] \\
& =\int_{0}^{\infty} \alpha h\left(\alpha \mid X_{1}, X_{2}, \cdots, X_{n}\right) d \alpha \\
& \tilde{\lambda}=E\left[h\left(\lambda \mid X_{1}, X_{2}, \cdots, X_{n}\right)\right] \\
& =\int_{0}^{\infty} \lambda h\left(\lambda \mid X_{1}, X_{2}, \cdots, X_{n}\right) d \lambda
\end{aligned}
$$

The Bayes risk of the parameters $\theta, \alpha$, and $\lambda$ based on square error loss function, respectively, are:

$$
\begin{aligned}
& \operatorname{var}(\tilde{\theta})=\int_{0}^{\infty} \frac{\int_{0}^{\infty} \frac{\int_{0}^{\infty} \pi\left(\theta \mid X_{1}, X_{2}, \cdots, X_{n}\right) d \alpha d \lambda}{\int_{0}^{\infty} \int_{0}^{\infty} \int_{0}^{\infty} \rho \theta^{\delta+1} \alpha^{\varepsilon+1} e^{-\eta \theta-\beta \theta}}\left(X_{1}, X_{2}, \cdots, X_{n}\right) d \Omega}{(\theta-\tilde{\theta}) d \theta(3.3 .12)} \\
& \operatorname{var}(\tilde{\alpha})=\int_{0}^{\infty} \frac{\int_{0}^{\infty} \int_{0}^{\infty} \pi\left(\alpha \mid X_{1}, X_{2}, \cdots, X_{n}\right) d \theta d \lambda}{\int_{0}^{\infty} \int_{0}^{\infty} \rho \theta_{0}^{\delta+1} \alpha^{\varepsilon+1} e^{-\eta \theta-\beta \theta}\left(X_{1}, X_{2}, \cdots, X_{n}\right) d \Omega}(\alpha-\tilde{\alpha}) d \alpha(3.3 .13) \\
& \operatorname{var}(\tilde{\lambda})=\int_{0}^{\infty} \frac{\int_{0}^{\infty} \int_{0}^{\infty} \pi\left(\lambda \mid X_{1}, X_{2}, \cdots, X_{n}\right) d \theta d \alpha}{\int_{0}^{\infty} \int_{0}^{\infty} \int_{0}^{\infty} \rho \theta^{\delta+1} \alpha^{\varepsilon+1} e^{-\eta \theta-\beta \theta}\left(X_{1}, X_{2}, \cdots, X_{n}\right) d \Omega}(\lambda-\tilde{\lambda}) d \lambda(3.3 .14)
\end{aligned}
$$

\subsection{Quasi-Bayesian Estimators}

The quasi-Bayesian estimation is similar to the quasilikelihood estimation in bath of them use the likelihood function, however, the earlier is different because it uses natural exponential of the quasi-likelihood function. For a sample of size $\mathrm{n}$ which is taken from the ELD, the natural exponential of the quasi-likelihood function is given by:

$$
\begin{aligned}
& Q^{*}(x, \theta, \alpha, \lambda)=e^{-\lambda \cdot \sum_{i=1}^{n} x_{i}-\left[\alpha\left[B\left(1-\frac{1}{\theta}, \alpha\right)-B(1, \alpha)\right]\right]} \\
& -\left[\frac{\alpha}{\lambda}\left[B\left(1-\frac{1}{\theta}, \alpha\right)-B(1, \alpha)\right]\right]^{n}
\end{aligned}
$$

By using the three prior distributions which discussed in (3.3.1), (3.3.2) and (3.3.3) for the parameters $\theta, \alpha$, and $\lambda$ respectively, then, the posterior distribution is:

$$
\begin{aligned}
& \pi^{*}\left(\Omega \mid X_{1}, X_{2}, \cdots, X_{n}\right)= \\
& \frac{\rho \theta^{\delta+1} \alpha^{\varepsilon+1} e^{-\eta \theta-\beta \theta} Q^{*}(x, \theta, \alpha, \lambda)}{\int_{0}^{\infty} \int_{0}^{\infty} \int_{0}^{\infty} \rho \theta^{\delta+1} \alpha^{\varepsilon+1} e^{-\eta \theta-\beta \theta} Q^{*}(x, \theta, \alpha, \lambda) d \Omega}
\end{aligned}
$$

Thus, the same technique for Bayesian estimation method from (3.3.6) to (3.3.14) and with the help of computer facilities will be used to evaluate the marginal distribution $h *\left(. \mid X_{1}, X_{2}, \cdots, X_{n}\right) \quad$, quasi-Bayesian estimators $\tilde{\theta}^{*}, \tilde{\alpha}^{*}$, and $\tilde{\lambda}^{*}$ under symmetric square loss function and Bayes risk for the parameters $\theta, \alpha$, and $\lambda$ respectively.

\section{Simulation Study}

The computer program MATHCAD (2001) is used to obtain numerical illustration for the last theoretical results for small, medium and large sample sizes. A comparison between the four estimation methods will be performed. 1000 samples generated from ELD with parameters $(\theta, \alpha, \lambda)$ are used at different values of these parameters. 
Mean square errors (MSE) of the three parameters will be calculated.

Table 1 indicates to that the quasi-likelihood and quasiBayesian estimators for the two parameters $\theta$ and $\alpha$ are better than the maximum likelihood and Bayesian - under symmetric square loss function estimators at all sample sizes respectively. Also, the performance of the quasilikelihood and quasi-Bayesian estimators for $\lambda$ are very close to the performance of the maximum likelihood and Bayesian - under symmetric square loss function estimators at all sample sizes respectively.

Through the results, we can see the mean square errors MSE of all estimations are decreasing as the size of sample is large. The quasi-Bayesian estimation is closest method because it goes to the real parameter values.

Table 1.

\begin{tabular}{|c|c|c|c|c|c|c|c|c|c|}
\hline $\mathrm{n}$ & parameter & MLE & MSE & QMLE & MSE & Bayes & MSE & QBayes & MSE \\
\hline \multirow{3}{*}{10} & $\theta$ & 0.618 & 0.278 & 0.255 & 0.024 & 0.508 & 0.277 & 0.134 & 0.024 \\
\hline & $\alpha$ & 0.397 & 0.431 & 0.808 & 0.034 & 0.287 & 0.43 & 0.687 & 0.034 \\
\hline & $\lambda$ & 1.016 & 0.045 & 1.11 & 0.021 & 0.906 & 0.044 & 0.989 & 0.021 \\
\hline \multirow{3}{*}{20} & $\theta$ & 0.49 & 0.182 & 0.237 & 0.011 & 0.38 & 0.181 & 0.116 & 0.011 \\
\hline & $\alpha$ & 0.551 & 0.315 & 0.819 & 0.017 & 0.441 & 0.314 & 0.698 & 0.017 \\
\hline & $\lambda$ & 0.932 & 0.019 & 1.106 & 0.019 & 0.822 & 0.018 & 0.985 & 0.019 \\
\hline \multirow{3}{*}{30} & $\theta$ & 0.459 & 0.155 & 0.224 & 0.001967 & 0.349 & 0.154 & 0.103 & 0.001967 \\
\hline & $\alpha$ & 0.585 & 0.291 & 0.829 & 0.005006 & 0.475 & 0.29 & 0.708 & 0.005006 \\
\hline & $\lambda$ & 0.903 & 0.013 & 1.104 & 0.018 & 0.793 & 0.012 & 0.983 & 0.018 \\
\hline \multirow{3}{*}{40} & $\theta$ & 0.372 & 0.088 & 0.222 & 0.0004727 & 0.262 & 0.087 & 0.101 & 0.0004727 \\
\hline & $\alpha$ & 0.681 & 0.2 & 0.831 & 0.00179 & 0.571 & 0.199 & 0.71 & 0.00179 \\
\hline & $\lambda$ & 0.869 & 0.012 & 1.103 & 0.017 & 0.759 & 0.011 & 0.982 & 0.017 \\
\hline \multirow{3}{*}{50} & $\theta$ & 0.278 & 0.035 & 0.224 & 0.0006819 & 0.168 & 0.034 & 0.103 & 0.0004669 \\
\hline & $\alpha$ & 0.828 & 0.124 & 0.832 & 0.001471 & 0.718 & 0.123 & 0.711 & 0.001471 \\
\hline & $\bar{\lambda}$ & 0.851 & 0.012 & 1.103 & 0.017 & 0.741 & 0.011 & 0.982 & 0.017 \\
\hline
\end{tabular}

\section{Conclusion}

This paper studied the estimation of parameters for the Exponentiated Lomax Distribution via four estimation method. These methods were maximum likelihood, quasilikelihood, Bayesian under symmetric square loss function and quasi-Bayesian estimations. Numerical study was investigated to illustrate the optimal procedure. When the sample sizes are increasing, the mean square errors MSE of all estimations are decreasing.

\section{References}

[1] Alzaatreh A., Lee C. \& Felix F. (2013). "A new method for generating families of continuous distributions". METRON. 71. 63-79.

[2] Amos D. (1983) "A portable Fortran subroutine for derivatives of Psi function". ACM Transactions on Mathematical software 9 (4). 494-502.

[3] Cordeiro M. \& Castro M. (2011). "A new family of generalized distributions". Journal of Statistical. Computation and Simulation. 81 (7). 883-898.

[4] Gauss M. \& Cordeiro M (2013). "The Exponentiated Generalized Class of Distributions". Journal of Data Science. 11. 1-12.

[5] Gupta C., Gupta P., \& Gupta D. (1998). "Modeling failure time data by Lehmann alternatives". Communications in StatisticsTheory and Methods. 27. 887-904.

[6] Nadarajah S. \& Kotz S. (2006). "The Exponentiated Type Distributions". Acta Applicandae Mathematica. 92 (2). 97-111.

[7] Salem H. (2013). "Inference on Stress-Strength Reliability for Weighted Weibull Distribution". American Journal of Mathematics and Statistics. 3 (4): 220-226.

[8] Wedderburn, R. (1974) "Quasi-Likelihood Functions, Generalized Models and the Gauss-Newton Method". Biometrika. 61 (3). 439443. 